Quim. Nova, Vol. 30, No. 8, 1900-1905, 2007

\title{
DESENVOLVIMENTO E VALIDAÇÃO DE METODOLOGIA DE ANÁLISE DE MISTURAS BIODIESEL:DIESEL UTILIZANDO CROMATOGRAFIA GASOSA-ESPECTROMETRIA DE MASSAS
}

\author{
Rita C. M. Faria, Michelle J. C. Rezende*, Claudia M. Rezende e Angelo C. Pinto \\ Instituto de Química, Universidade Federal do Rio de Janeiro, Centro de Tecnologia, Bloco A, Ilha do Fundão,
} 21949-900 Rio de Janeiro - RJ, Brasil

Recebido em 13/11/06; aceito em 21/6/07; publicado na web em 9/11/07

\begin{abstract}
DEVELOPMENT AND VALIDATION OF A METHODOLOGY FOR ANALYSIS OF BIODIESEL: DIESEL BLENDS USING GAS CHROMATOGRAPHY-MASS SPECTROMETRY. The aim of this work was to develop and validate a method of analysis of biodiesel:diesel blends using HRGC-MS in SIM mode. The proposed method is applicable to biodiesel from linoleic oils, such as soybean oil. The following results were obtained: linearity over the methyl linoleate concentration range of $250-2500 \mathrm{mg} \mathrm{L}^{-1}\left(\mathrm{R}^{2}>0.999\right)$; limits of detection and quantification of 2 and $5 \mathrm{mg} \mathrm{L}^{-1}$, respectively; recovery from 82 to $96 \%$ of biodiesel in diesel and repeatability $<2 \%$ (RSD). Comparison between two operators showed that it is not necessary the same operator to do the analysis.
\end{abstract}

Keywords: biodiesel; diesel; HRGC-MS-SIM.

\section{INTRODUÇÃO}

A maior parte de toda energia consumida no mundo provém do petróleo, carvão e gás natural. Essas fontes são limitadas e com previsão de esgotamento no futuro. A busca por fontes alternativas de energia torna-se, assim, de suma importância.

A utilização de biodiesel como combustível vem apresentando potencial promissor no mundo inteiro. Em primeiro lugar, pela sua enorme contribuição ao meio ambiente, com a redução qualitativa e quantitativa dos níveis de poluição ambiental. Em segundo lugar, como fonte estratégica de energia renovável em substituição ao óleo diesel. No cenário brasileiro, o seu uso pode também reduzir a dependência de importação deste combustível.

O óleo diesel é o principal produto do fracionamento do petróleo no Brasil. Esse combustível é constituído por hidrocarbonetos parafínicos, olefínicos e aromáticos com cadeia carbônica de 6 a 30 átomos. Alguns compostos apresentam também enxofre, oxigênio e nitrogênio. Já o biodiesel é constituído por ésteres alquílicos obtidos, principalmente, a partir da transesterificação de óleos vegetais ou gordura animal. A reação é conduzida na presença de metanol ou etanol e de um catalisador adequado. Este biocombustível também pode ser produzido a partir da esterificação direta de ácidos graxos livres. Entretanto, este processo é raramente utilizado uma vez que as fontes de triglicerídeos são mais disponíveis do que de ácidos graxos ${ }^{1}$. O biodiesel possui algumas vantagens sobre o diesel, tais como alto número de cetano; alto ponto de fulgor; menor emissão de material particulado, monóxido de carbono e dióxido de carbono; biodegradabilidade; além de ser isento de enxofre e de compostos aromáticos ${ }^{2,3}$.

Em 2004, foi lançado no Brasil o Programa Nacional de Produção e Uso de Biodiesel. A lei no 11.097 , de 13 de janeiro de 2005, dispõe sobre a introdução do biodiesel na matriz energética brasileira. A lei determina a adição mínima obrigatória de 5\% de biodiesel ao óleo diesel a partir de janeiro de 2013, e estabelece a utilização de um percentual mínimo obrigatório intermediário de $2 \%$ a partir de janeiro de 2008. A Agência Nacional de Petróleo, Gás Natural e Biocombustíveis (ANP), órgão regulador, aponta para a necessidade de se avaliar a qualidade desta mistura a partir da realização de aná-

*e-mail: michelle@iq.ufrj.br lises laboratoriais e emissão de Boletim de Conformidade. O desenvolvimento de metodologias para análise de misturas biodiesel:diesel passa a ser um recurso estratégico no controle de qualidade do combustível a ser disponibilizado nos postos de abastecimento.

Dentre as ferramentas analíticas disponíveis para identificação e quantificação de compostos em misturas, pode-se destacar a Cromatografia Gasosa de Alta Resolução acoplada à Espectrometria de Massas (CGAR-EM), a Espectroscopia na Região do Infra-Vermelho (IV) e do Ultra-Violeta (UV) e a Ressonância Magnética Nuclear (RMN). Em agosto de 2006 foi realizada uma consulta à base de dados "Sci Finder" a fim de verificar a existência de trabalhos voltados para a aplicação destas técnicas na análise de misturas biodiesel:diesel. O acesso forneceu aproximadamente 1830 referências utilizando biodiesel como tópico de pesquisa. Em seguida, a ferramenta de refino foi aplicada neste conjunto de referências utilizando, separadamente, "chromatography", "infrared", "nmr" e "ultra violet" como palavras-chave. O resultado da pesquisa selecionou 69, 26, 18 e 15 referências para cada palavra-chave, respectivamente. A partir da leitura cuidadosa deste conjunto de referências pôde-se constatar que somente 4 publicações tinham como objetivo analisar misturas biodiesel-diesel. Um artigo utilizou a cromatografia líquida com detetor $\mathrm{UV}^{4}$, dois trabalhos empregaram a técnica de $\mathrm{IV}^{5-6}$ e o último comparou a técnica de IV com $\mathrm{RMN}^{7}$. Com relação às demais publicações, nenhuma teve como objetivo central a análise de biodiesel em diesel, a grande maioria utilizava estas técnicas para o acompanhamento da produção de biodiesel.

O objetivo deste trabalho foi o desenvolvimento e a validação de uma metodologia de análise de misturas biodiesel:diesel empregando CGAR-EM no modo de análise por monitoramento seletivo de íons (MSI). Além do controle de qualidade do combustível, esta metodologia pode ser empregada no estudo de estabilidade da mistura e das condições adequadas de estocagem.

\section{PARTE EXPERIMENTAL}

\section{Materiais e reagentes}

Linoleato de metila e 1-pentadecanol foram provenientes da Aldrich (> 99\%, EUA). Cloreto de propanoíla e sílica (70-230 mesh) 
foram adquiridos da Merck (EUA). Carbonato de potássio, sulfato de sódio e os solventes (hexano, acetato de etila, diclorometano e metanol) foram adquiridos da Vetec (Brasil). O diesel foi comprado em posto de abastecimento de combustível e o óleo de soja foi comprado em estabelecimento comercial.

\section{Síntese e purificação do padrão interno}

O propanoato de $n$-pentadecanoíla foi o composto selecionado para uso como padrão interno (PI) na quantificação das misturas biodiesel:diesel por CGAR-EM-MSI. A síntese do éster foi realizada a partir da esterificação de cloreto de propanoíla com 1pentadecanol em diclorometano. A mistura foi mantida em agitação constante a temperatura ambiente por $90 \mathrm{~min}$. O solvente foi evaporado sob pressão reduzida e o produto foi purificado em coluna de sílica usando hexano como eluente. A pureza do padrão interno (> 99\%) foi determinada por cromatografia gasosa de alta resolução com detector de ionização por chama (CGAR-DIC).

\section{Preparo da curva de calibração}

Foram construídas duas curvas de calibração para o linoleato de metila, sendo cada uma realizada por um analista diferente (Analista A e Analista B). Cada curva foi constituída por seis pontos e cada ponto foi realizado em triplicata. As amostras padrão foram preparadas a partir de um volume conhecido $(25,50,75,100,150$ e $250 \mu \mathrm{L}$ ) da solução estoque de linoleato de metila $5000 \mathrm{mg} \mathrm{L}^{-1}, 50$ $\mu \mathrm{L}$ de diesel e $100 \mu \mathrm{L}$ da solução estoque de padrão interno 2000 $\mathrm{mg} \mathrm{L^{-1 }}$. O volume final foi completado até $500 \mu \mathrm{L}$ com acetato de etila. As amostras padrão apresentaram concentração de 250, 500, $750,1000,1500$ e $2500 \mathrm{mg} \mathrm{L}^{-1}$ de linoleato de metila, respectivamente, e $400 \mathrm{mg} \mathrm{L}^{-1}$ do padrão interno.

\section{Instrumentação e condições cromatográficas}

As análises por CGAR-EM foram realizadas em cromatógrafo Agilent modelo 6890 acoplado a um detetor de espectrometria de massas Agilent modelo 5973 com ionização por impacto de elétrons a $70 \mathrm{eV}$ e analisador do tipo quadrupolo. A coluna capilar utilizada foi DB-1 HT (J\&W) $15 \mathrm{~m}$ x 0,25 mm x 0,10 $\mu \mathrm{m}$ de espessura de fase. $\mathrm{O}$ gás de arraste utilizado foi hélio $\left(1,7 \mathrm{~mL} \mathrm{~min}^{-1}\right)$ e a pressão na cabeça da coluna foi de 8 psi. O volume de injeção da amostra foi 1,0 $\mu \mathrm{L}$ com divisão de fluxo de 1:20. A temperatura inicial do forno foi de $80{ }^{\circ} \mathrm{C}$ seguida por três rampas de aquecimento: a primeira taxa de aquecimento foi de $10^{\circ} \mathrm{C} \min ^{-1}$ até $150{ }^{\circ} \mathrm{C}$; a segunda, de 5 ${ }^{\circ} \mathrm{C} \min ^{-1}$ até $200{ }^{\circ} \mathrm{C}$ e a última, de $15{ }^{\circ} \mathrm{C} \min ^{-1}$ até $300{ }^{\circ} \mathrm{C}$ permanecendo nesta temperatura por $5 \mathrm{~min}$. O tempo total de corrida foi 28,70 min. O injetor foi operado a $290{ }^{\circ} \mathrm{C}$ e a interface a $300{ }^{\circ} \mathrm{C}$. As medidas quantitativas foram realizadas no modo MSI. Os íons monitorados e os tempos de retenção dos compostos estão apresentados na Tabela 1. O modo SCAN, na faixa de massa de 40 a 500 Daltons, foi utilizado para confirmação dos espectros de massas.

Tabela 1. Tempos de retenção $\left(t_{R}\right)$ e íons selecionados

\begin{tabular}{lcc}
\hline Compostos & $\mathrm{t}_{\mathrm{R}}(\mathrm{min})$ & Íons característicos $(\mathrm{m} / \mathrm{z})$ \\
\hline $\begin{array}{l}\text { Propanoato de } \\
n \text {-pentadecanoíla }\end{array}$ & 10,30 & $182 / 210 / \mathbf{2 5 5}$ \\
Linoleato de metila & 11,49 & $220 / 262 / \mathbf{2 6 3}$ \\
\hline
\end{tabular}

Íons para análise quantitativa em negrito.

\section{Avaliação de interferentes}

Para verificar possíveis interferentes no método proposto, a matriz diesel foi diluída em acetato de etila na razão 1:10 e, em seguida, analisada por CGAR-EM no modo MSI. Foram monitorados os íons de $m / z 182,210$ e 255, referentes ao propanoato de $n$-pentadecanoíla, e de $m / z, 220,262$ e 263, referentes ao linoleato de metila.

\section{Preparo das misturas biodiesel:diesel para o estudo de recuperação}

O biodiesel foi preparado a partir da reação de transesterificação do óleo de soja com metanol na razão molar óleo:álcool 1:9 e utilizando $0,5 \%(\mathrm{~m} / \mathrm{m})$ de carbonato de potássio como catalisador. A mistura foi mantida sob refluxo e agitação constante por $3 \mathrm{~h}$. Os ésteres metílicos foram isolados após neutralização do meio reacional com ácido clorídrico diluído, seguida de extração com acetato de etila e lavagens com água destilada. A fase orgânica foi tratada com sulfato de sódio anidro, filtrada e, em seguida, evaporada. A conversão em ésteres metílicos (99\%) foi calculada a partir dos espectros de Ressonância Magnética Nuclear de Hidrogênio (RMN de $\left.{ }^{1} \mathrm{H}\right)^{8}$.

Para o estudo de recuperação, foram analisadas três concentrações diferentes de biodiesel em diesel (B0,7, B1,9 e B4,3). Foram selecionadas concentrações próximas aos limites inferior e superior da faixa de estudo estabelecida e uma concentração próxima ao percentual mínimo obrigatório intermediário de biodiesel em diesel (B2). Para cada concentração foram preparadas três misturas de biodiesel:diesel. Cada amostra analisada foi composta por 50 $\mu \mathrm{L}$ da mistura correspondente, $100 \mu \mathrm{L}$ da solução estoque de padrão interno $2000 \mathrm{mg} \mathrm{L}^{-1}$ e $350 \mu \mathrm{L}$ de acetato de etila. Foram preparadas 9 amostras e, cada uma, foi analisada em triplicata. Vale ressaltar que as concentrações selecionadas para o estudo da recuperação foram diferentes das concentrações estabelecidas para construção das curvas de calibração.

\section{RESULTADOS E DISCUSSÃO}

\section{Desenvolvimento do método cromatográfico}

Em virtude da complexidade da matriz diesel, o método foi desenvolvido no modo de análise por monitoramento seletivo de íons. Esta técnica é freqüentemente aplicada para misturas de compostos onde é requerida uma análise quantitativa mais sensível e específica9 ${ }^{9}$

O éster linoleato de metila (LIN) foi selecionado para o monitoramento e quantificação do biodiesel presente nas misturas biodiesel:diesel. O ácido linoleico está presente majoritariamente em diversos óleos vegetais, dentre eles, óleo de soja (53-55\%), óleo de milho (57-60\%), óleo de algodão (55-57\%) e óleo de girassol (68$73 \%)^{10-12}$. Esta foi a principal motivação para a escolha deste éster no desenvolvimento deste primeiro estudo.

A quantificação foi realizada pelo método de padronização interna, empregando propanoato de $n$-pentadecanoíla como padrão interno. Este composto é diferente de qualquer éster graxo oriundo de óleo vegetal ${ }^{13}$. Adicionalmente, possui boa estabilidade e tempo de retenção distinto do analito, ou seja, do linoleato de metila. A partir dos espectros de massas do linoleato de metila e do propanoato de $n$-pentadecanoíla, apresentados na Figura 1, foram selecionados três íons característicos de cada éster para caracterização. Após esta seleção, os picos correspondentes ao padrão interno e ao linoleato de metila foram confirmados pela injeção em diesel, em diferentes concentrações. A Tabela 1 apresenta os tempos de retenção, 
de acordo com a programação de temperatura adotada, e os fragmentos característicos selecionados para as análises qualitativas e quantitativas.

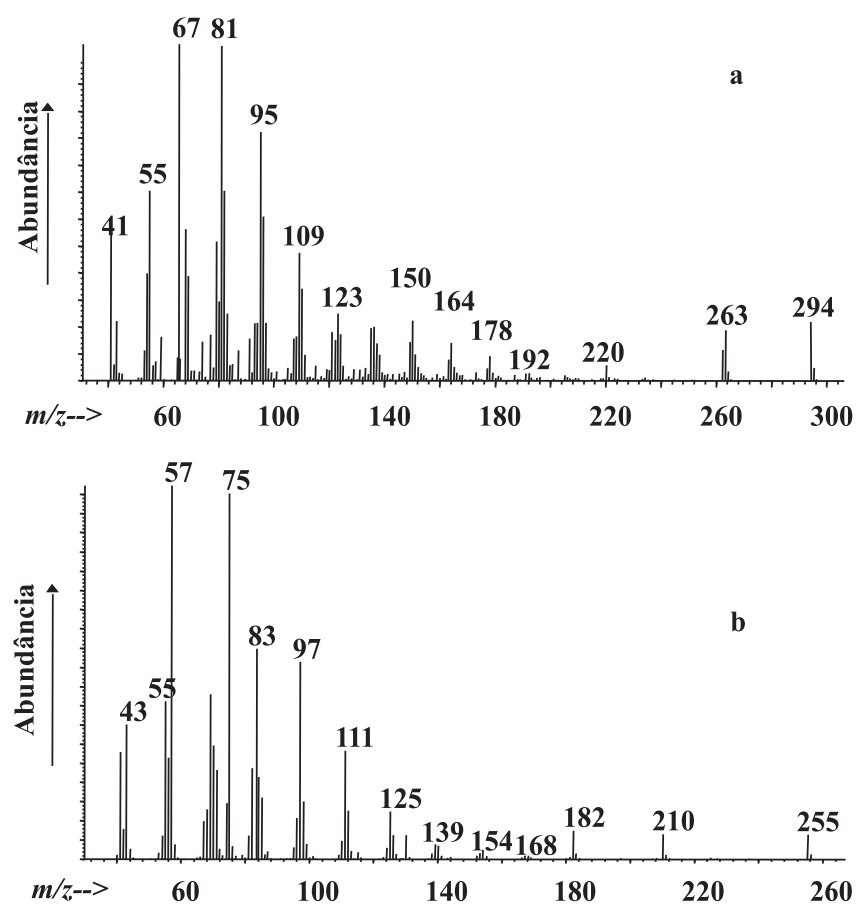

Figura 1. Espectros de massas: a) linoleato de metila e b) propanoato de n-pentadecanoíla (padrão interno)

\section{Validação do método proposto}

A validação do método foi realizada a partir da análise dos seguintes critérios de desempenho: análise de interferentes, linearidade, limites de detecção e quantificação, exatidão e precisão. Os testes estatísticos utilizados incluíram a análise de resíduo, os testes de Grubbs, de Cochran e o teste F de Snedecor. A exatidão foi avaliada por ensaios de recuperação e a precisão foi avaliada por estudo de repetitividade e precisão intermediária a partir da comparação entre a inclinação das retas obtidas por dois analistas. Este estudo foi realizado baseando-se nas orientações sobre validação descritas por Ribani et $a l^{14}$ e Fernandes ${ }^{15}$. A seguir serão apresentados os resultados de cada critério de desempenho.

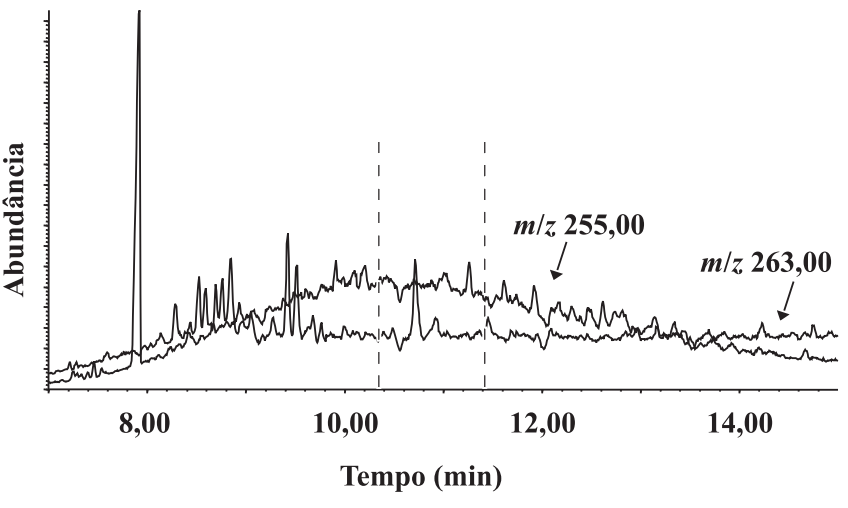

Figura 2. Cromatograma CGAR-EM-MSI m/z,255 e 263 do diesel

Avaliação de interferentes

Para avaliar a presença de interferentes, o diesel puro foi analisado por CGAR-EM-MSI. O objetivo desta caracterização foi detectar, na matriz, compostos que pudessem apresentar os íons selecionados para quantificar o linoleato de metila e o padrão interno. A Figura 2 apresenta o cromatograma dos íons $\mathrm{m} / \mathrm{z}, 255$ e 263 do diesel analisado.

$\mathrm{O}$ cromatograma mostra que o diesel não possui interferentes nos tempos de retenção (linhas tracejadas) do propanoato de n-pentadecanoíla e do linoleato de metila. Desta forma, pôde-se assegurar que os registros dos íons de $\mathrm{m} / z 255$ e 263 nos cromatogramas das misturas biodiesel:diesel não pertencem ao diesel, mas sim aos ésteres em questão.

\section{Determinação da faixa de trabalho}

A seleção da faixa de trabalho foi realizada com base na proposta do governo brasileiro de implementação da mistura B2 (2\% de biodiesel em diesel) como percentual mínimo obrigatório intermediário. A faixa de trabalho selecionada foi de 250 a $2500 \mathrm{mg} \mathrm{L}^{-1}$ de linoleato de metila. Nesta faixa, o método permite a quantificação de 0,5 a $5 \%$ de biodiesel.

\section{Avaliação da linearidade}

As curvas de calibração dos analistas A e B foram construídas a partir de 6 pontos em triplicata, nas concentrações de 250 a 2500 $\mathrm{mg} \mathrm{L}^{-1}$ de linoleato de metila e $400 \mathrm{mg} \mathrm{L}^{-1}$ do padrão interno em diesel. A Figura 3 mostra os cromatogramas dos íons $m / z 255$ e 263 da mistura biodiesel:diesel em três concentrações.

Uma seqüência de ajustes e testes estatísticos foi aplicada aos

Tabela 2. Resultado do teste de Cochran para o analista A

\begin{tabular}{lcccccc}
\hline & LIN 250 & LIN 500 & LIN 750 & LIN 1000 & LIN 1500 & LIN 2500 \\
\hline Áreas & 0,8803 & 1,6919 & 2,4540 & 3,1487 & 4,7224 & 7,6055 \\
& 0,9009 & 1,6428 & 2,4583 & 3,1670 & 4,6748 & 7,6844 \\
& 0,8914 & 1,6702 & 2,4627 & 3,1648 & 4,6856 & 7,6264 \\
$<\mathrm{x}_{1}>$ & 0,8909 & 1,6683 & 2,4583 & 3,1602 & 4,6943 & 7,6388 \\
$\mathrm{~S}$ & $1,0310 \mathrm{E}-02$ & $2,4605 \mathrm{E}-02$ & $4,3501 \mathrm{E}-03$ & $9,9912 \mathrm{E}-03$ & $2,4955 \mathrm{E}-02$ & $4,0878 \mathrm{E}-02$ \\
$\mathrm{~S}^{2}$ & $1,0630 \mathrm{E}-04$ & $6,0541 \mathrm{E}-04$ & $1,8923 \mathrm{E}-05$ & $9,9823 \mathrm{E}-05$ & $6,2277 \mathrm{E}-04$ & $1,6710 \mathrm{E}-03$ \\
DPR & 1,1573 & 1,4749 & 0,1770 & 0,3162 & 0,5316 & 0,5351 \\
\hline
\end{tabular}

$\Sigma \mathrm{S}^{2} \quad 0,0031 ; \mathrm{C}_{\mathrm{cal}} \quad 0,1993 ; \mathrm{C}_{\mathrm{tab}} \quad 0,6160$

$<\mathrm{x}_{1}>$ : valor médio; $\mathrm{S}$ : desvio padrão; $\mathrm{S}^{2}$ variância; $\Sigma \mathrm{S}^{2}$ : somatório das variâncias; $\mathrm{DPR}$ : desvio padrão relativo; $\mathrm{C}_{\text {cal }}$ e $\mathrm{C}_{\text {tab }}$ : valor calculado e tabelado, respectivamente, de Cochran (95\% de confiança). 
Tabela 3. Resultado do teste de Cochran para o analista B

\begin{tabular}{lcccccc}
\hline & LIN 250 & LIN 500 & LIN 750 & LIN 1000 & LIN 1500 & LIN 2500 \\
\hline Áreas & 0,8977 & 1,6943 & 2,4792 & 3,2939 & 4,7466 & 7,7554 \\
& 0,8888 & 1,7048 & 2,5185 & 3,2438 & 4,7722 & 7,7483 \\
& 0,8715 & 1,7384 & 2,5044 & 3,2276 & 4,7645 & 7,7332 \\
$<\mathrm{x}_{1}>$ & 0,8860 & 1,7125 & 2,5007 & 3,2551 & 4,7611 & 7,7456 \\
$\mathrm{~S}$ & $1,3323 \mathrm{E}-02$ & $2,3036 \mathrm{E}-02$ & $1,9910 \mathrm{E}-02$ & $3,4564 \mathrm{E}-02$ & $1,3134 \mathrm{E}-02$ & $1,1338 \mathrm{E}-02$ \\
$\mathrm{~S}^{2}$ & $1,7749 \mathrm{E}-04$ & $5,3067 \mathrm{E}-04$ & $3,9639 \mathrm{E}-04$ & $1,1947 \mathrm{E}-03$ & $1,7251 \mathrm{E}-04$ & $1,2854 \mathrm{E}-04$ \\
DPR & 1,5037 & 1,3452 & 0,7962 & 1,0619 & 0,2759 & 0,1464 \\
\hline
\end{tabular}

$\Sigma \mathrm{S}^{2} \quad 0,0026 ; \mathrm{C}_{\mathrm{cal}} \quad 0,4595 ; \mathrm{C}_{\text {tab }} \quad 0,6160$

$<\mathrm{x}_{1}>$ : valor médio; $\mathrm{S}$ : desvio padrão; $\mathrm{S}^{2}$ variância; $\Sigma \mathrm{S}^{2}$ : somatório das variâncias; DPR: desvio padrão relativo; $\mathrm{C}_{\text {cal }}$ e $\mathrm{C}_{\text {tab }}$ : valor calculado e tabelado, respectivamente, de Cochran ( $95 \%$ de confiança).

dados experimentais obtidos. Os dados foram ajustados ao modelo de regressão linear $(y=a+b x)$, sendo submetidos ao teste de Grubbs, que se destina a verificar valores aberrantes. De acordo com o teste, nenhum valor foi descartado com $95 \%$ de confiança. Em seguida, as curvas de calibração foram submetidas ao teste de Cochran para avaliar o desvio bilateral das variâncias a um nível de

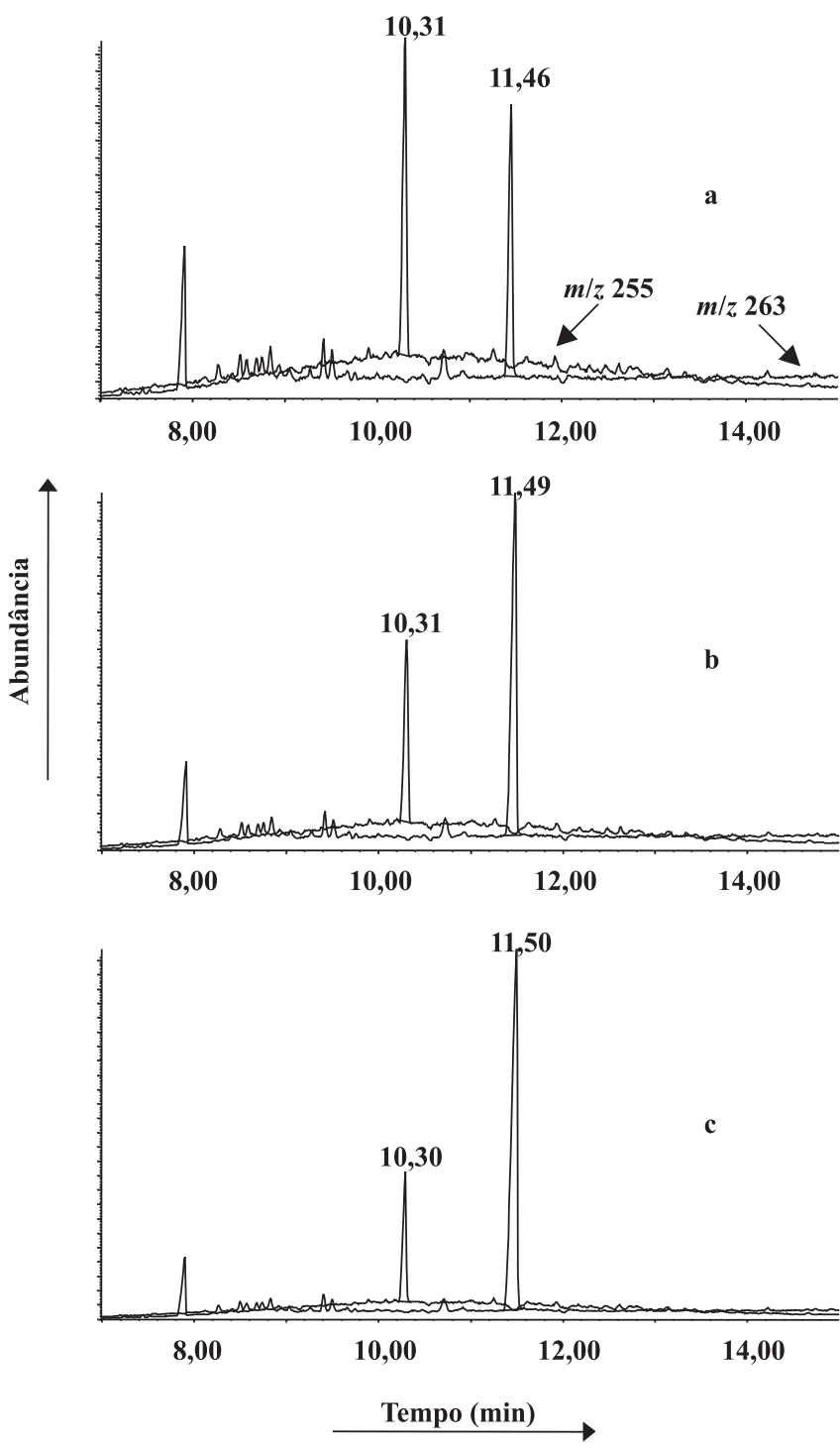

Figura 3. Cromatograma de íons totais CGAR-EM-MSI m/z, 255 e 263 em três concentrações de LIN em diesel: a) $250 \mathrm{mg} \mathrm{L}^{-1}$; b) $750 \mathrm{mg} \mathrm{L}^{-1}$; c) $1500 \mathrm{mg} \mathrm{L}^{-1}$; PI a $400 \mathrm{mg} \mathrm{L^{-1 }}$ significância de 5\%. Este teste destina-se a avaliar a homogeneidade das variâncias, o que permite utilizar a curva de calibração obtida com um limite de confiança aceitável. Os resultados estão apresentados nas Tabelas 2 e 3 para as curvas obtidas pelos analistas A e B, respectivamente.

Os valores de Cochran calculados foram inferiores ao valor de Cochran tabelado, mostrando variâncias homogêneas conforme o aumento da concentração. Este resultado indica que ao longo da faixa de concentração estudada o modelo tem um comportamento homocedástico. Como o teste mostrou que as variâncias são estatisticamente equivalentes, as curvas de calibração foram determinadas através de regressão linear. A Tabela 4 apresenta as equações das retas e os valores de $\mathrm{R}^{2}$ obtidos para cada analista.

Tabela 4. Parâmetros das curvas de calibração dos analistas A e B

\begin{tabular}{lcc}
\hline Analista & Equação & $\mathrm{R}^{2}$ \\
\hline $\mathrm{A}$ & $\mathbf{Y}=1,2484 \mathbf{X}+0,1753$ & 0,9998 \\
$\mathrm{~B}$ & $\mathbf{Y}=1,2653 \mathbf{X}+0,1896$ & 0,9997 \\
\hline
\end{tabular}

$\mathbf{Y}=$ área LIN/área PI; $\mathbf{X}=$ concentração LIN/concentração PI.

Para verificar a eficiência do modelo de regressão linear proposto foram analisados os gráficos de resíduo das duas curvas de calibração. O resíduo foi calculado através da diferença entre o valor observado e o valor calculado pela equação da reta de regressão para cada valor de concentração de linoleato de metila. Esta análise corroborou a conclusão do teste de Cochran, que indicou o método como homocedástico na faixa de concentração estudada.

Determinação dos limites de detecção e quantificação

Os limites de detecção (LD) e quantificação (LQ) foram calculados pelo método da relação sinal-ruído. A relação sinal-ruído utilizada para o cálculo do LD e do LQ foi 3:1 e 10:1, respectivamente. Os limites de detecção e quantificação fornecidos pela análise foram de 2 e $5 \mathrm{mg} \mathrm{L}^{-1}$ de linoleato de metila, respectivamente. Considerando que o diesel foi diluído em uma razão 1:10 para minimizar o efeito da matriz, o limite de quantificação do método corresponde a uma concentração de linoleato de metila de $50 \mathrm{mg}$ $\mathrm{L}^{-1}$ na mistura biodiesel:diesel.

A quantificação do biodiesel presente no diesel, entretanto, é realizada em função do óleo vegetal empregado para sua produção, ou seja, com base na concentração de linoleato de metila presente na matéria-prima.

Avaliação da exatidão por estudo de recuperação

A recuperação do método foi avaliada a partir de amostras preparadas com biodiesel de óleo de soja misturado ao óleo diesel nas 
concentrações 0,$7 ; 1,9$ e 4,3\%. Para cada concentração foram preparadas três amostras e estas foram analisadas em triplicata.

O biodiesel foi preparado a partir da transesterificação de óleo de soja usando excesso de metanol e carbonato de potássio como catalisador ${ }^{16}$. A conversão de $99 \%$ de triglicerídeos em ésteres metílicos foi determinada por RMN de ${ }^{1} \mathrm{H}$. A análise do biodiesel por CGAR-DIC mostrou a presença de $57,2 \%$ de linoleato de metila no biocombustível.

A partir do método desenvolvido foi determinada a concentração de linoleato de metila presente em cada amostra. Considerando que o éster compreende $57,2 \%$ do biodiesel utilizado no preparo das amostras, foi calculada a quantidade do biocombustível presente nas mesmas. A Tabela 5 apresenta os resultados do estudo de recuperação.

Tabela 5. Resultados da análise de exatidão por recuperação

\begin{tabular}{|c|c|c|c|c|c|c|}
\hline Mistura & $\mathrm{A}_{1}$ & $<\mathrm{A}_{1}>$ & $\mathrm{B}_{\text {teor }}$ & $\mathrm{B}_{\exp }$ & $\begin{array}{c}\text { Rend. } \\
(\%)\end{array}$ & $\begin{array}{l}\text { Erro } \\
(\%)\end{array}$ \\
\hline B0,7 & $\begin{array}{l}1,3308 \\
1,3485 \\
1,3757\end{array}$ & 1,3517 & 0,75 & 0,72 & 96,0 & 4,0 \\
\hline B0,7 & $\begin{array}{l}1,3086 \\
1,3080 \\
1,2996\end{array}$ & 1,3054 & 0,73 & 0,69 & 94,5 & 5,5 \\
\hline B 0,7 & $\begin{array}{l}1,3574 \\
1,3678 \\
1,3536\end{array}$ & 1,3596 & 0,76 & 0,72 & 94,7 & 5,3 \\
\hline B1,9 & $\begin{array}{l}3,2317 \\
3,3140 \\
3,3305\end{array}$ & 3,2921 & 1,87 & 1,78 & 95,2 & 4,8 \\
\hline B1,9 & $\begin{array}{l}3,2703 \\
3,3151 \\
3,3268\end{array}$ & 3,3041 & 1,92 & 1,79 & 93,2 & 6,8 \\
\hline B 1,9 & $\begin{array}{l}3,2104 \\
3,2447 \\
3,3263\end{array}$ & 3,2605 & 1,92 & 1,76 & 91,7 & 8,3 \\
\hline $\mathrm{B} 4,3$ & $\begin{array}{l}6,4691 \\
6,5590 \\
6,5496\end{array}$ & 6,5259 & 4,27 & 3,55 & 83,1 & 16,9 \\
\hline $\mathrm{B} 4,3$ & $\begin{array}{l}6,4461 \\
6,4412 \\
6,4693\end{array}$ & 6,4522 & 4,28 & 3,51 & 82,0 & 18,0 \\
\hline $\mathrm{B} 4,3$ & $\begin{array}{l}6,5332 \\
6,4621 \\
6,5001\end{array}$ & 6,4985 & 4,29 & 3,54 & 82,5 & 17,5 \\
\hline
\end{tabular}

* $\mathrm{A}_{1}$ : área LIN/área PI; $<\mathrm{A}_{1}>$ : média das áreas LIN/área PI; $\mathrm{B}_{\text {teor }}$ : concentração de biodiesel teórica da mistura; $\mathrm{B}_{\text {exp }}$ : concentração de biodiesel experimental da mistura; Rend.: rendimento.

O erro relativo encontrado variou de 4 a $18 \%$ ficando abaixo de $20 \%$ para todas as concentrações analisadas. A recuperação variou de 82 a $96 \%$. Para a amostra de $1,9 \%$ em particular, o erro relativo foi inferior a $10 \%$. Assim, pode-se concluir que o método de quantificação proposto apresentou uma recuperação adequada na concentração próxima à concentração de biodiesel em diesel determinada pelo governo $(2 \%)$.

\section{Avaliação da precisão por estudo de repetitividade e precisão intermediária}

A precisão foi avaliada em função da repetitividade e precisão intermediária. Para avaliar a precisão do método proposto, dois analistas (A e B), em dias diferentes, realizaram, no mesmo aparelho, o procedimento para construção de duas curvas de calibração na mesma faixa de concentração.
A repetitividade foi avaliada a partir da estimativa do desvio padrão relativo (DPR) para cada analista. Os resultados estão apresentados nas Tabelas 2 e 3, já citadas anteriormente. Verificou-se uma boa repetitividade, considerando os dados de cada analista isoladamente. Pode-se observar que o desvio padrão relativo obtido pelo analista A foi inferior a $2 \%$, em toda faixa de trabalho. $\mathrm{O}$ mesmo comportamento foi observado para o analista B, onde o desvio também foi inferior a $2 \%$.

Para verificar a precisão intermediária do método, foi feito um estudo comparativo das inclinações das retas obtidas pelos analistas A e B. Para esta avaliação foi realizado o Teste de hipóteses (F de Snedecor), que avalia o desvio bilateral das variâncias a um nível de significância de 5\%. O objetivo deste estudo foi verificar se o método fornece os mesmos resultados para analistas diferentes (Tabela 6). O resultado mostrou que as retas dos analistas A e B possuem inclinações iguais, não sendo necessária a construção de uma curva de calibração diária e nem de um mesmo analista para a realização das análises.

Tabela 6. Resultado do estudo comparativo entre as inclinações das retas obtidas pelos analistas A e B

\begin{tabular}{|c|c|c|c|c|}
\hline Analista & $\mathrm{N}$ & Equações & $\mathrm{R}$ & $\mathrm{Se}^{2}$ \\
\hline A & 6 & $\begin{array}{c}\mathbf{Y}=1,2484 \mathbf{X} \\
+0,1753\end{array}$ & 0,9998 & 0,004439 \\
\hline B & 6 & $\begin{array}{c}\mathbf{Y}=1,2653 \mathbf{X} \\
+0,1896\end{array}$ & 0,9997 & 0,006856 \\
\hline
\end{tabular}

$\mathrm{F}_{\text {cal }}=1,544 \quad \mathrm{~F}_{\mathrm{tab}}=5,820$

$\mathrm{t}_{\mathrm{cal}}=-0,71 \quad \mathrm{t}_{\mathrm{tab}}=2,31$

* N: número de pontos da reta; R: coeficiente de correlação linear da reta; $\mathrm{Se}^{2}$ : variância da reta; $\mathrm{F}$ : valor de Snedecor; t: valor de tStudent a um nível de significância de $5 \%$.

\section{CONCLUSÃO}

Uma metodologia para a quantificação de misturas biodiesel:diesel por CGAR-EM-MSI foi desenvolvida e validada. A metodologia desenvolvida é aplicada para biodiesel oriundo de óleos ricos em ácido linoleico. Como perspectiva, a metodologia será ampliada para biodiesel produzido a partir de outros óleos vegetais de interesse nacional.

Os critérios de desempenho avaliados foram linearidade, limites de detecção e quantificação, exatidão e precisão. A faixa de concentração estudada foi de 250 a $2500 \mathrm{mg} \mathrm{L}^{-1}$ de linoleato de metila. O método mostrou-se adequado uma vez que o teor de biodiesel nas misturas biodiesel:diesel a serem analisadas mantémse acima do limite de quantificação encontrado. Além disto, o método de quantificação proposto apresentou uma boa recuperação, visto que para uma mistura de biodiesel:diesel de 1,9\%, o erro relativo foi inferior a $10 \%$. No estudo de repetitividade, o desvio padrão relativo manteve-se inferior a $2 \%$ em toda a faixa de trabalho para os analistas A e B, indicando uma boa sensibilidade e precisão dos resultados. Adicionalmente, a comparação entre a inclinação das retas obtidas pelos dois analistas mostrou que não é necessária a construção de uma curva de calibração diária e nem de um mesmo analista para a realização das análises.

\section{AGRADECIMENTOS}

Ao Programa de Formação de Recursos Humanos da ANP; ao Laboratório de RMN do Instituto de Química da UFRJ; à D. R. Fernandes pela colaboração no desenvolvimento deste trabalho. 


\section{REFERÊNCIAS}

1. Pinto, A. C.; Guarieiro, L. L. N.; Rezende, M. J. C.; Ribeiro, N. M.; Torres, E. A.; Lopes, W. A.; Pereira, P. A. P.; de Andrade, J. B.; J. Braz. Chem Soc. 2005, 16, 1313.

2. Haas, M. J.; Scott, K. M.; Alleman, T. L.; McCormick, R. L.; Energy Fuels 2001, 15, 1207

3. Krawczyk, T.; Inform. 1996, 7, 801

4. Foglia, T. A.; Jones, K. C.; Phillips, J. G.; Chromatographia 2005, 62, 115.

5. Pimentel, M. F.; Ribeiro, G. M. G. S.; Cruz, R. S.; Stragevitch, L.; Pacheco Filho, J. G. A.; Teixeira, L. S. G.; Microchem. J. 2006, 82, 201.

6. Oliveira, J. S.; Montalvão, R.; Daher, L.; Suarez, P. A. Z.; Rubim, J. C.; Talanta 2006, 69, 1278

7. Knothe, G.; J. Am. Oil Chem. Soc. 2001, 78, 1025.
8. Gelbard, G.; Brés, O.; Vargas, R. M.; Vielfaure, F.; Schuchardt, U. F.; $J$ Am. Oil Chem. Soc. 1995, 72, 1239.

9. Barker, J.; Mass Spectrometry, $2^{\text {nd }}$ ed., ACOL Serie John Wiley \& Sons: Chichester, 1999

10. Goering, C. E.; Schwab, A. W.; Daugherty, M. J.; Pryde, E. H.; Heakin, A. J.; Trans. ASAE 1982, 25, 1472.

11. Kincs, F. R.; J. Am. Oil Chem. Soc. 1985, 62, 815.

12. Babayan, V. K.; Lipids 1987, 22, 417.

13. Rezende, M. J. C.; Perruso, C. R.; Azevedo, D. A.; Pinto, A. C.; J. Chromatogr., A 2005, 1063, 211.

14. Ribani, M.; Bottoli, C. B. G.; Collins, C. H.; Jardim, I. C. S. F.; Melo, L. F. C.; Quim. Nova. 2004, 27, 771.

15. Fernandes, D. R.; Tese de Mestrado, Universidade Federal do Rio de Janeiro, Brasil, 2004

16. Schuchardt, U.; Sercheli, R.; Vargas, R. M.; J. Braz. Chem. Soc. 1998, 9 , 199. 